\title{
ESTUDIO RANDOMIZADO SOBRE CONTINENCIA URINARIA POSTPROSTATECTOMÍA RADICAL CON REHABILITACIÓN PERINEAL KIESICA PREVIA
}

\author{
Ignacio Tobía, Mariano S. González, Pablo Martínez, Juan C. Tejerizo, Guillermo Gueglio,
} Oscar Damia, María I. Martí y Carlos A. Giudice.

Servicio de Urología. Hospital Italiano de Buenos Aires. Buenos Aires. República Argentina.

\begin{abstract}
Resumen.- OBJETIVO: La incontinencia urinaria es una de las principales complicaciones luego de la realización de prostatectomía radical (PR). La rehabilitación kinésica preoperatoria, podría ser de utilidad como tratamiento preventivo de esta complicación. Demostrar la utilidad de la kinesiología perineal preoperatoria en la recuperación precoz de la continencia urinaria post prostatectomía radical.
\end{abstract}

MÉTODOS: Ensayo Clínico Controlado Randomizado Aleatorizado. 38 pacientes fueron divididos en dos grupos de 19 previo a la realización de la PR. El primer grupo (K) recibió tratamiento kinésico preoperatorio, mientras que el segundo grupo (NK) no (grupo control). Se evaluó la continencia de orina a los 14, 30 y 60 días post extracción de sonda.
RESULTADOS: No hubo diferencias epidemiológicas y de biología tumoral entre grupos. El porcentaje de pacientes continentes en el grupo $\mathrm{K}$ al los $14,30 \mathrm{y}$ 60 días, respectivamente fue de $47,36 \%, 47,36 \%$ y $78,9 \%$, respectivamente, mientras que en el grupo NK fueron de $47,36 \%, 47,36 \%$ y $89,4 \%$, respectivamente (p>0,05).

CONCLUSIÓN: Los ejercicios kinésicos perineales previos a la prostatectomía radical, no disminuyeron los tiempos de recuperación de la continencia urinaria ni la ocurrencia de la misma.

Palabras clave: Prostatectomía radical. Incontinencia urinaria. Kinesioterapia pelviana.

Summary.- OBJECTIVES: Urinary incontinence is one of the main complications after radical prostatectomy (RP). Preoperative kinetic physiotherapy could be useful as a preventive treatment of these complication. The objective of this work is to demonstrate the usefulness of preoperative perineal kinetic physiotherapy for early recovery of urinary continence after radical prostatectomy.

METHODS: Randomized controlled clinical trial. 38 patients were divided into groups of 19 before radical prostatectomy. The first group $(K)$ received preoperative kinesic treatment whereas the second group (NK) did not (control group). Urinary continence was evaluated at 74,30 and 60 days after catheter retrieval.

RESUITS: There were not epidemiological or tumor biology differences between groups. The percentages of continent patients in group $K$ at 14,30 and 60 days 
were $47.36 \%, 47.36 \%$ and $78.9 \%$ respectively, whereas in the NK group where $47.36 \%, 47.36 \%$, and $89.4 \%$ respectively ( $p>0.05$ ).

CONCLUSIONS: Kinesic perineal exercises before radical prostatectomy did not diminish the times of urinary continence recovery or its appearance.

Keywords: Radical prostatectomy. Urinary incontinence. Pelvic kinesic physiotherapy.

\section{INTRODUCCIÓN}

El cáncer de próstata es la segunda causa de muerte oncológica en el hombre, luego del cáncer de pulmón (1-2).

Existen diferentes opciones terapéuticas para el tratamiento de la enfermedad diagnosticada en estadios localizados. La prostatectomía radical es uno de los tratamientos más recomendados, ya que otorga mejores posibilidades desde el punto de vista oncológico con respecto a otros tratamientos (3-4).

Las complicaciones postoperatorias más frecuentes de esta técnica son la estrechez de anastomosis uretro-vesical, la impotencia sexual, y la incontinencia de orina. Las dos últimas reducen su incidencia si durante la cirugía se preservan las bandeletas neurovasculares adosadas a la cara pósteroinferior prostática.

La incontinencia de orina presenta una incidencia de 6-87\%, según las series (5-7). Esta variación está influida por los distintos parámetros y las diferentes definiciones utilizadas por los autores, nomenclatura que está en constante revisión. Si bien pocos pacientes quedan con incontinencia definitiva y socialmente inhabilitante, muchos pasan meses hasta su resolución espontánea. Poder explicarle al paciente cuando podrá, estimativamente, dejar de usar apósitos o pañales $y$, por lo tanto, recuperar su vida laboral y social es el objetivo de estos estudios.

Las terapéuticas utilizadas para este tipo de incontinencia son la farmacológica, la quirúrgica y la rehabilitación kinésica.

Varios trabajos evalúan la efectividad de los ejercicios kinésicos perineales posteriores a la prostatectomía radical, con resultados muy variables. Casi todos los trabajos describen pocos cambios en cuanto a los resultados de continencia finales, pero sí un logro mas rápido de la misma $(8,9)$.
En el hombre son reconocibles dos zonas responsables de la continencia: la primera es el esfínter uretral proximal, incluyendo al cuello vertical, la glándula prostática y la uretra prostática hasta el veru montanum. La segunda es el esfínter uretral distal desde el veru montanum hasta la uretra bulbar. Este último esfínter posee una parte intrínseca (rabdoesfínter) capaz de mantener el tono uretral por largos periodos de tiempo, una segunda parte compuesta por músculo estriado, extrínseca, que suplementa a la anterior ante situaciones de estrés, y una tercera parte compuesta por músculo liso, intrínseca, continuación de la capa superficial del detrusor (10).

Durante una prostatectomía radical el esfínter uretral proximal es removido o dañado en gran parte, lo mismo pasa con el veru montanum y el ápex prostático, por lo tanto, la continencia queda exclusivamente a cargo de el esfínter distal (11). Esta zona es el lugar donde actúa la kinesioterapia perineal postoperatoria, mediante el fortalecimiento del mecanismo de continencia. De este modo podría esperarse similar resultado con dicho tratamiento, pero utilizado en forma previa a la cirugía.

Nuestro objetivo es evaluar si la realización de kinesiología perineal preoperatoria logra una recuperación precoz de la continencia urinaria post prostatectomía radical.

\section{MATERIAL Y MÉTODO}

Entre los meses de enero de 2006 y agosto de 2007 se realizaron 80 prostatectomías radicales como tratamiento de adenocarcinoma de próstata localizado, en el Hospital Italiano de Buenos Aires. Del total, 38 pacientes fueron seleccionables para nuestro estudio según criterios de inclusión.

Se randomizó a los pacientes en dos grupos de 19. El grupo intervención (K), realizó tratamiento kinésico preoperatorio y el grupo control (NK), no.

Todos los procedimientos quirúrgicos fueron iguales: prostatectomía radical con linfadenectomía iliobturatriz por vía suprapúbica, con disección de pico a base prostática y fueron realizadas por los mismos cirujanos (C.G y P.M.)

Los criterios de inclusión pre quirúrgicos fueron: edad entre 45 y 75 años, PSA menor o igual de 20, Score combinado de Gleason en la punción biopsia preoperatoria menor o igual a 8 , estadio clínico T1 o T2 según la clasificación de la A.J.C.C. de 1997 (12). Los criterios de inclusión intra quirúrgicos fueron: ausencia de complicaciones intra operatorias 
como sangrado y que por lo tanto se pudo realizar una buena disección del pico prostático y al menos una bandeleta neurovascular conservada.

No se incluyeron pacientes con fístulas, hematomas, sangrados o reoperaciones.

Ningún paciente presentaba incontinencia de orina clínica previo al procedimiento.

Los ejercicios kinésicos fueron todos realizados por la misma kinesióloga.

\section{Metodología de la Kinesiología Perineal:}

El objetivo de la kinesiología perineal fue fortalecer el piso pélvico, aumentar la compliance vesical y favorecer la sinergia vésico-esfinteriana.

Se llevó a cabo en 4 fases:

Fase 1 Reconocimiento de la función esfinteriana.

Fase 2 Fortalecimiento del piso pélvico.

Fase 3 Automatización de la sinergia vésico-esfinteriana.

Fase 4 Incorporar estos valores en lo cotidiano y ante situaciones de mayor exigencia.

La metodología fue:

1- Evaluación del piso pélvico: Se realizó a través del tacto rectal con paciente en posición supina, con piernas flexionadas y en abducción. La escala de medición abarcó un rango de 0 a 5, que va desde una ausencia de contracción, a una contracción máxima.

2- Medición del valor muscular: Con aparatología capaz de graficar mediante un programa la fuerza muscular. Se utilizó un perinómetro, que determina calidad, cantidad y nivel de fatiga de la musculatura. Además este diseño de aparato sirvió para entrenar la función muscular. El gráfico que se obtiene se usa para objetivar la evolución y que pueda ser controlado por el médico.

\section{3- Electroestimulación de baja y mediana frecuencia} neo-fadica: Se realizó electroestimulación, combinada con ejercicios para estimular las fibras tónicas y fáscicas, para cada caso en particular, potenciando los resultados. La aplicación de este tipo de corriente apunta a reforzar la musculatura perineal, inhibir las contracciones no inhibidas del detrusor, y en consecuencia mejorar la compliance vesical y la flexibilidad de la uretra.
4- Plan de ejercicios en domicilio: El paciente practicó con una frecuencia que varió entre 7 a 10 veces por día, lo aprendido en consultorio.

En todos los casos se evaluó la continencia a los 14, 30 y 60 días posteriores a la extracción de la sonda, que en todos los casos se retiró a los 21 días.

La clasificación de la incontinencia urinaria se realizó mediante evaluación clínica, y se definió como incontinente aquel que requirió la utilización de por lo menos 1 paño (13).

Para la comparación estadística se utilizó Ttest para edad y Wilcoxon para PSA, Gleason Pre y post. La comparación de continencia en el tiempo fue evaluada mediante Chi cuadrado.

\section{RESULTADOS}

Se evaluaron 38 pacientes. La edad media fue de 62,68 (r:49-75). En el grupo de tratamiento kinésico previo (K) fue de 63,10 (r: $49-75)$, y en los no tratado previamente (NK) 62,26 (r: 51-71).

EI PSA medio fue de $8,32 \mathrm{ng} / \mathrm{ml}$ en el grupo $\mathrm{K}$ (r: 4,37-17) y de $10,54 \mathrm{ng} / \mathrm{ml}$ en el grupo NK (r: 2,5-20).

El promedio d score de Gleason combinado de la punción biopsia prostática fue de 6,47 (r:5-8) en el grupo K y 6,31 (r:4-8) en el NK. El promedio de Gleason combinado de la pieza operatoria fue de 6,84 (r:6-9) y 6,84 (r:5-10) para los grupos NK y K, respectivamente.

No hubo diferencias estadísticamente significativas para edad $(p=0,674)$, PSA $(p=0.208)$, Gleason pre $(p=0,762)$, ni Gleason postoperatorio $(p=$ $0,824)$.

Para comparación en la evaluación de la continencia se compararon ambos grupos a los 14, 30 y 60 días, luego de la extracción de la sonda.

A los 14 días no se encontraron diferencias entre los grupos, solo $9(47,36 \%)$ pacientes del grupo $\mathrm{K}$ se encontraban continentes y 9 del grupo NK $(47,36 \%)$, claramente no existe diferencia estadísticamente significativa.

A los 30 días se encontraron los mismos porcentajes de continencia.

Luego de 60 días, 15 pacientes $(78,9 \%)$ se encontraban continentes del grupo K y $17(89,4 \%)$ 
del grupo NK, no encontrando diferencias estadísticamente significativas $(p=0,3736)$ entre los grupos y siendo, llamativamente mayor la continencia en el grupo que no realizó kinesiología previa.

\section{DISCUSIÓN}

La incontinencia urinaria es una de las principales complicaciones posteriores a la prostatectomía radical, la cual repercute en las esferas física, psíquica y social del paciente. Esta complicación se halla presente en 6 a $87 \%$ de los pacientes sometidos a este tipo de cirugías (5-7). Las diferencias en las cifras pueden atribuirse a las diferentes definiciones de incontinencia urinaria y al modo de medirla y evaluarla.

Los factores de riesgo de incontinencia post prostatectomía no están claramente establecidos. Suelen mencionarse la edad, el tamaño y la configuración prostática y del tumor, la presencia de obstrucción vesical y/o disfunción vesical preoperatorios y factores relacionados con la técnica quirúrgica como la lesión de las bandeletas neurovasculares y la preservación del cuello vesical. En un estudio multivariado de la Universidad de Baylor, los factores de riesgo significativos para incontinencia de orina persistente post prostatectomía radical fueron mayor edad del paciente, desarrollo de estrechez de la anastomosis uretrovesical, reoperaciones endoscópicas y técnica quirúrgica utilizada por el cirujano (14). Con respecto a la vía de abordaje, no parece haber diferencia entre los índices de continencia de la vía abierta versus vía laparoscópica (15).

Existen pocos trabajos en la literatura que evalúen tratamientos preoperatorios para mejorar la continencia.

Es sabido que luego de la prostatectomía radical la continencia depende de la estabilidad vesical y del funcionamiento del esfínter interno. Con respecto a este último factor está bien establecido que un porcentaje alto de pacientes incontinentes luego de cirugía presentan potenciales de denervacion del esfínter periuretral (16). Podría esperarse que el fortalecimiento de dicho esfínter mediante ejercicios perineales pudiera disminuir la chance de aparición de esta complicación. Sin embargo estudios urodinámicos realizados a pacientes sometidos a este tipo de cirugía, apuntando a estos factores conjuntamente con otros parámetros de importancia, demuestran que estas podrían no ser las únicas causas de incontinencia post prostatectomía (17-18).

Con respecto a la preservación del cuello vesical existen sobradas muestras en la literatura a cerca de la falta de mejoría en la continencia postoperatoria con este tipo de medidas. Como ejemplo sólo citaremos a autores como Licht, Braslis y Lowe, quienes lo demuestran fehacientemente (19-21).

Varios investigadores creen que la disminución de la compliance vesical y la inestabilidad de la misma es la principal causa de la incontinencia postprostatectomia (22).

Sin embargo Oelrich demostró que con la edad el esfínter estriado en el hombre progresivamente es invadido por tejido conectivo con lo que disminuye su contracción y por ende la continencia (23).

Las terapéuticas para la incontinencia post prostatectomía, van desde el tratamiento médico hasta el quirúrgico, contando con la posibilidad del tratamiento kinésico. Dentro de este se encuentran el biofeedback, los ejercicios de los músculos de la pelvis y la reeducación de la micción (24).

Si bien en sus cifras de pacientes continentes a fin del estudio no se hallan diferencias entre grupos, Parekh describe una recuperación de la continencia urinaria más rápida con el ejercicio de los músculos del piso de la pelvis, acelerando la reinserción social del paciente (25).

Rodrigues Netto sugiere, en caso de indicar ejercicios kinésicos, hacerlo en el postoperatorio y en forma temprana para mejorar los resultados (26).

En un ensayo randomizado, Franke y Smith no encontraron efectos beneficiosos significativos con el uso del biofeedback temprano posterior a la prostatectomía (86 vs. $88 \%$ respectivamente) (27).

Asimismo, varios autores recomiendan comenzar con ejercicios preoperatorios para ayudar a los pacientes a conocer el piso pélvico $(28,29)$.

Nuestro grupo encontró una mejoría en la continencia desde los 14 días post extracción de sonda hasta los 60 días con porcentajes similares a los descriptos por otros autores, no existiendo los beneficios esperados de la kinesioterapia perineal. Dentro de estas presentaciones hallamos a Bales, quien estudia el tratamiento kinésico pre y postoperatorio y muestra resultados muy similares de continencia a los 6 meses comparando pacientes tratados con entrenamiento y biofeedback antes y después de la cirugía versus un grupo control (94 vs. $96 \%$ respectivamente). Este autor concluye que los ejercicios preoperatorios no mejoran el tiempo ni la calidad de la continencia posterior a la prostatectomía radical (5). Tres grupos 
de trabajo europeos llegan a la misma conclusión, y agregan que no realizarlos puede ahorrar hasta 711 euros por paciente sin cambios en el resultado $(8,30,31)$.

En una revisión de trials realizada por un grupo canadiense, sobre continencia pos cirugía prostática oncológica, sólo hallan algún beneficio cuando se realizan ejercicios perineales durante el tiempo existente hasta la extracción de sonda, aunque estos beneficios no son del todo concluyentes, opinión que concuerda con Franke et al, quienes no hallan beneficios con este tipo de tratamientos (27-32).

Sueppel y cols. tomaron dos grupos, a uno le dieron instrucciones de cómo realizar los ejercicios kinésicos y biofeedback previos a la cirugía y otro grupo se le dieron instrucciones de cómo realizarlos con posterioridad a la misma, encontrando mejoría en los síntomas, disminución del número de apósitos por día y mejor calidad de vida en el primer grupo, sin embargo los porcentajes a fin de estudio llegan a ser bastante similares (33).

Si bien la mayoría de los estudios presentados varían en porcentajes y de alguna manera se diferencian al nuestro, todos llegamos a la misma conclusión: - los grupos que realizan algún tipo de kinesioterapia y los que no la realizan no presentan grandes diferencias en cuanto al porcentaje de pacientes continentes dentro del primer año post cirugía, con tiempos similares de recuperación.

\section{CONCLUSIÓN}

Los ejercicios kinésicos perineales previos a la prostatectomía radical, no mejoraron los resultados ni disminuyen los tiempos de recuperación de la continencia urinaria, así como tampoco reducen la ocurrencia de la misma.

\section{BIBLIOGRAFÍA Y LECTURAS RECOMENDADAS ( ${ }^{*}$ lectura de interés $y^{* *}$ lectura fundamental)}

*1. SILVERBERG, E.; BORING, C.C.; SQUIRES, T.S.: "Cancer statistics". CA, 40: 9, 1990.

*2. BEGUN, F.P.: "Epidemiología e historia natural del cáncer de próstata". Lepor/Lawson: Enfermedades de la próstata, Editorial Panamericana: 313327, 1994.
3. MYERS, R.P.: "Radical Prostatectomy: Making it a better operation in the new millennium". Int. J. Urol., 8: 9, 2001.

*4. VASHI, A.R.; OESTERLING, J.E.: "Radical retropubic prostatectomy for localizated prostate cancer". Kaisaj A.V., Murphy G.P., Denis L., Griffith K., editors. Textbook of prostate cancer: $\mathrm{Pa}-$ thology, diagnosis and treatment. London: Martin Dunitz, 189, 2001.

5. BALES, G.T.; GERBER, G.S.; MINOR, T.X. y cols.: "Effect of preoperative biofeedback/pelvis floor training on continence in men undergoing radical prostatectomy". Urology, 56: 627, 2000.

6. RUDY, D.C.; WOODSIDE, J.R.; CRAWFORD, E.D.: "Urodynamic evaluation of incontinence in patients undergoing modified Campbell radical retropubic prostatectomy: A prospective study". J. Urology, 132: 708, 1984.

*7. STEINER, M.S.; MORTON, R.A.; WALSH, P.C.: "Impact of anatomical radical prostatectomy on urinary continence". J. Urology, 1145: 512, 1991.

8. WILLE, S.; SOBOTTKA, A.; HEIDENREICH, A. y cols.: "Pelvic floor exercises, electrical stimulation and biofeedback after radical prostatectomy: Results of a prospective randomized trial". J. Urology, 170: 490, 2003.

**9. VAN KAMPEN, M.; DE WEERDT, W.; VAN POPPEL, H. y cols.: "Effect of pelvic floor reeducation on duration and degree of incontinence after radical prostatectomy: A randomized controlled trial". Lancet, 355: 98, 2000.

**10. TURNER-WARWICK, R.: "The sphincter mechanisms. Their relation to prostatic enlargement and its treatment". Hinman F. Jr.(ed): Benign Prostatic Hypertrophy. New York, Springer. Verlag, Pag. 809, 1983.

11. ZIMMERN, P. E.; LEACH, G.E.: "Treatment of incontinence in men". Semin. Urol., 7: 124, 1989.

12. AMERICAN JOINT COMMITEE ON CANCER 1997.: “A.J.C.C. Cancer Staging Manual”. 5th edition. Lippimcott-Raven Eds. Pag. 220-223, 1997.

13. JONLER, M.; MADSEN, F.A.; RHODES, P.R. y cols.: "A prospective study of quantification of urinary incontinence and quality of life in patients undergoing radical retropubic prostatectomy". Urology, 48: 433, 1996.

14. EASTHAM, J.; GOAD, J.R.; ROGERS, E. y cols.: "Risk factors for urinary incontinence after radical retropubic prostatectomy". J. Urol., 156: 1707, 1996.

**15. JACOBSEN, N.E.; MOORE, K.N.; ESTEY, E. y cols.: "Open versus laparoscopic radical prostatectomy: A prospective comparison of postoperative urinary incontinence rates". J. Urol., 177: $615,2007$. 
16. SALINAS CASADO, J.; VIRSEDA CHAMORRO, M.; FERNANDEZ AJUBITA, H. y cols.: "Urodynamics in urinary incontinence post radical prostatectomy". Arch. Esp. Urol., 51: 801, 1998.

17. FICAZZOLA, M.A.; NITTI, V.W.: "The etiology of post-radical prostatectomy incontinence and correlation of symptoms with urodynamic findings". J. Urol., 160: 1317, 1998.

18. GUDZIAK, M.R.; Mc GUIRE, E.J.; GORMLEY, E.A.: "Urodynamic assessment of urethral sphincter function in post-prostatectomy incontinence". J. Urol., 156: 1131, 1996.

19. LICHT, M.R.; KLEIN, E.A.; TUASON, L. y cols.: "Impact of bladder neck preservation during radical prostatectomy on continence and cancer control". Urology, 44: 883, 1994.

20. BRASLIS, K.G.; PETSCH, M.; LIM, A. y cols.: "Bladder neck preservation following radical prostatectomy: Continence and margins". Eur. Urol., 28: 202, 1995.

**21. LOWE, B.A.: "Comparison of bladder neck preservation to bladder neck resection in maintaining postprostatectomy urinary incontinence". Urology, 48: 889, 1996.

22. LEACH, G.E.; YIP, C.M.; DONOVAN, B.J.: "Post-prostatectomy incontinence: The influence of bladder disfunction”. J. Urol., 138: 574, 1987.

23. OELRICH, T.M.: "The urethral sphincter muscle in the male". Am. J. Anat., 158: 229, 1980.

24. MOUL, J.W.: "For incontinence after prostatectomy tap a diversity of treatments". Contemp. Urol., 6: 78, 1994.

**25. PAREKH, A.R.; FENG, M.I.; KIRAGES, D. y cols.: "The role of pelvis exercises on post-pros- tatectomy incontinence”. J. Urology, 170: 130, 2003.

**26. TARCIA KAHIHARA, C.; FERRERIRA, U.; NARDI PEDRO, R. y cols.: "Early versus delayed physiotherapy in the treatment of post-prostatectomy male urinary incontinence". Arch. Esp. Urol., 59: 773, 2006.

*27. FRANKE, J.J.; GILBERT, W.B.; GRIER, J. y cols.: "Early post-prostatectomy pelvic floor biofeedback". J. Urology, 163: 191, 2000.

28. GIBOD, L.E.: "Incontinence after radical prostatectomy". European Urology Today. Proceeding from EUF Symposium.

29. MOUL, J.W.: "Pelvic muscle rehabilitation in males following prostatectomy". National Association for continence, 17: 1, 1999.

30. LILLI, P.; MERCURIALI, M.; FIORI, M. y cols.: "Impact of preoperative biofeedback on incontinence in cancer patients undergoing radical prostatectomy". Arch. Ital. Urol. Androl., 78: 92, 2006.

*31. CORNEL, E.B.; DE WIT, R.; WITJES, J.A.: "Evaluation of early pelvic floor physiotherapy on the duration and degree of urinary incontinence after radical retropubic prostatectomy in a non-teaching hospital". World J. Urol., 23: 353, 2005.

*32. HUNTER, K.F.; MOORE, K.N.; CODY, D.J. y cols.: "Conservative management for postprostatectomy urinary incontinence". Cochrane Database Syst. Rev., 2: CD001843, 2004.

33. SUEPPEL, C.; KREDER, K.; SEE, W.: "Improved conrtinence outcomes with preoperative pelvis floor muscle strengthening exercises". Urologic Nursing, 21: 201, 2001. 